\title{
Energy-Aware Underwater Optical System with Combined Solar Cell and SPAD Receiver
}

\author{
Sahar Ammar, Osama Amin, Mohamed-Slim Alouini and Basem Shihada
}

\begin{abstract}
This letter designs a simultaneous lightwave and power transfer (SLIPT) system in the underwater environment using the power splitting technique. The data is sent by a laser diode and received by a multi-element receiver composed of a Single Photon Avalanche Diode (SPAD) and a solar cell that decodes the transmitted symbols and harvests electrical energy from the optical signal, respectively. In this context, we propose an energy-aware SLIPT system that optimizes the power split between the receivers and the SPAD detecting threshold to maximize the harvested energy while considering minimum link quality for the communication service. Although the optimization problem is not convex, we derive its feasibility conditions in terms of the system's parameters and propose an optimal resource allocation algorithm. Finally, we introduce some numerical results to investigate the effect of SPAD's dead time and other parameters on the system's performance.
\end{abstract}

Index Terms-Underwater Optical Wireless Communication (UOWC), Simultaneous Lightwave Information and Power Transfer (SLIPT), Single Photon Avalanche Diode (SPAD), Solar Cell, Energy Harvesting, Throughput.

\section{INTRODUCTION}

With almost three-quarters of the planet's surface covered by water, various human activities have always been carried out in this environment, from marine life exploration and climate change studies to oil monitoring and military operations. Hence, there is a need for an efficient and flexible communication solution for underwater wireless communication. Underwater Optical Wireless Communication (UOWC) is shown to be a good candidate that provides high data rates for medium ranges compared to both acoustic and radio frequency communication systems [1]-[4]. However, the UOWC systems face several challenges, such as the high absorption and attenuation, light beam alignment between transceivers, and the common problem of accessing energy supplies in the marine environment.

Simultaneous Lightwave Information and Power Transfer (SLIPT) is a technology proposed to enable both communication and energy harvesting from optical signals [5], [6]. Hence, it can support systems with limited energy supplies, especially underwater, such as underwater Internet-of-Things devices, autonomous self-powered machines, and remote sensors. SLIPT can be established through time switching, power splitting, and spatial splitting techniques [7]. Thanks to their ability to perform both tasks, solar panels are most suitable to design SLIPT systems operating over a limited link range without external power supply. In this regard, a large active area solar panel is used to establish an UOWC lens-free system capable of simultaneously harvest energy and decode

The authors are with CEMSE Division, King Abdullah University of Science and Technology (KAUST), Thuwal, Makkah Province, Saudi Arabia. Email: \{sahar.ammar, osama.amin, slim.alouini, basem.shihada\}@kaust.edu.sa. information from the transmitted optical waves while easing the alignment requirements [7]-[9]. Specifically, the authors of [7], [8] illustrate experiments for underwater self-powered systems by a solar panel that switches its operation to shift between the two operational modes. In [9], an optimization study based on time switching between both modes in solar cells is developed to maximize the energy harvesting subject to a minimum error probability. However, using solar cells at optical receivers limits significantly the communication performance due to its low bandwidth. Multi-element receiver is a promising approach to improve the operation of different modes similar to the combined solar-cell and photo-diode receiver proposed for indoor visible light communication systems [6]. From detection prospective, Single Photon Avalanche Diodes (SPADs) are characterized by their high detection efficiency [10], low power consumption, and insensitivity to light during the dead time induced by the quenching process. The latter can be active (AQ) or passive (PQ), where AQ SPADs have shorter constant dead times and higher count rates than PQ SPADs [11]. Another way to improve the optical data rate is using symbols with unequal probability to improve a performance of optical communication systems [12].

This paper adopts an underwater power-split-based SLIPT system to provide reliable communication and energy harvesting services. To the best of our knowledge, this is the first research work that uses a combined solar cell and SPAD receiver supporting these services. Furthermore, we employ unequal probability symbols to generalize the study and improve the system's performance. Moreover, we propose an energy-aware scheme to maximize the harvested energy while satisfying a robust communication link with a minimum throughput requirement by tuning the power splitting factor and the SPAD threshold. Finally, we present numerical results showing the optimization feasibility region and receiver performance in terms of harvested energy and throughput performance while investigating the effect of SPAD's dead time and different system parameters. The rest of this paper is organized as follows, Section II provides a description of the underwater SLIPT system model. Section III deals with the design of the Energyaware system maximizing the harvested energy according to the power splitting ratio for specific throughput performance. Finally, Section IV discusses the numerical results followed by the paper conclusion in Section V.

\section{SySTEM DESCRIPTION}

Throughout this section, we present the proposed underwater SLIPT system, starting with the transmission signal model, passing by the appropriate channel model, and ending with the receiver model followed by the definition of the adopted performance metrics. 


\section{A. Transmitter model}

We consider a laser diode in the visible range and employ the on off keying modulation signal with a bit duration of $T_{\mathrm{b}}$ carried over a direct current (DC) signal such that the optical transmitted signal $s_{\mathrm{t}}(t)$ is given by

$$
s_{\mathrm{t}}(t)=P_{\mathrm{s}}[D+m(t)],
$$

where $P_{\mathrm{s}}$ is the laser source optical power, $D$ is the DC bias, and $m(t)$ denotes the transmitted signal, defined as follows

$$
m(t)=\left\{\begin{array}{cc}
P_{\mathrm{A}} & \text { bit }=1 \\
-P_{\mathrm{A}} & \text { bit }=0
\end{array},\right.
$$

where the probabilities of sending " 0 " and " 1 " bits are $p_{0}$ and $p_{1}$, respectively, and $P_{\mathrm{A}}$ is the peak amplitude expressed as

$$
P_{\mathrm{A}}=\left\{\begin{array}{ll}
D-I_{\mathrm{L}} & \text { if } D<\left(I_{\mathrm{L}}+I_{\mathrm{H}}\right) / 2 \\
I_{\mathrm{H}}-D & \text { if } D \geq\left(I_{\mathrm{L}}+I_{\mathrm{H}}\right) / 2
\end{array},\right.
$$

where $I_{\mathrm{L}}$ and $I_{\mathrm{H}}$ are the minimum and maximum diode input bias current.

\section{B. Channel model}

The UOWC is achieved by a line of sight (LOS) link that is subject to several attenuation and fading factors. First, we use the following distance-based path loss model $L$ [13],

$$
L=\left(\frac{D_{\mathrm{R}}}{\theta d}\right)^{2} e^{-c_{\mathrm{ex}} d\left(\frac{D_{\mathrm{R}}}{\theta d}\right)^{T}},
$$

where $d$ is the link distance, $D_{\mathrm{R}}$ is the receiver aperture diameter, $\theta$ denotes the full width transmitter beam divergence angle, $T$ is a correction coefficient and $c_{\mathrm{ex}}$ is the extinction coefficient. As for the turbulence-induced fading coefficient $\alpha$, which captures also the effect of air bubbles and effect of temperature gradient, we use the mixture Exponential and Generalized Gamma (EGG) distribution model with the following probability density function [14],

$$
f_{\alpha}(\alpha)=\omega f(\alpha ; \mu)+(1-\omega) g(\alpha ;|a, b, c|),
$$

where $f(\alpha ; \mu)$ is an Exponential distribution function with a parameter $\mu$ and is written as follows

$$
f(\alpha ; \mu)=\frac{1}{\mu} \exp \left(-\frac{\alpha}{\mu}\right)
$$

and $g(\alpha ;[a, b, c])$ is the Generalized Gamma distribution with parameters $a, b$ and $c$, and is defined as [14]

$$
g(\alpha ;[a, b, c])=c \frac{\alpha^{a c-1}}{b^{a c}} \frac{\exp \left(-\left(\frac{\alpha}{b}\right)^{c}\right)}{\Gamma(a)} .
$$

In (5), $\omega$ represents the distributions mixture coefficient where $0<\omega<1$. As a result, we define the aggregate UOWC channel as $h(d)=L(d) \alpha$.

\section{Receiver model}

We assume a power split-based SLIPT system with a splitting factor $\beta \in[0,1]$. The receiver is composed of an AQ SPAD to detect data and a solar cell used only to harvest energy where the power splitting can be achieved by different methods such as using an optical beamsplitter and tunable lenses. Firstly, the photon counting process, in presence of dead time $\tau$, during the time interval $\left(0, T_{\mathrm{b}}\right)$ is modeled by the following distribution [11],

$$
\begin{gathered}
p\left(k, r_{i}\right)=\sum_{n=0}^{k} \psi\left(n, r_{i}\left(T_{\mathrm{b}}-(k+1) \tau\right)\right) \\
-\sum_{n=0}^{k-1} \psi\left(n, r_{i}\left(T_{\mathrm{b}}-k \tau\right)\right), \text { for } k<k_{\max },
\end{gathered}
$$

where $r_{i}$ denotes the average photon arrival rate given a transmitted symbol $i=0,1, k_{\max }=\left\lfloor T_{\mathrm{b}} / \tau\right\rfloor+1$ is the maximum number of total counted photons and $\psi(n, x)=\frac{x^{n}}{n !} e^{-x}$.

Thus, the average received SPAD signal, in the presence of dead time, can be written as follows

$$
\bar{y}_{\mathrm{SPAD}}=\left\{\begin{array}{ll}
\lambda_{1}=r_{1}\left(1-\left(\lambda_{\mathrm{th}}+1\right) \frac{\tau}{T_{\mathrm{b}}}\right) T_{\mathrm{b}}, & i=1 \\
\lambda_{0}=r_{0}\left(1-\left(\lambda_{\mathrm{th}}+1\right) \frac{\tau}{T_{\mathrm{b}}}\right) T_{\mathrm{b}}, & i=0
\end{array},\right.
$$

where $\lambda_{\text {th }}$ denotes the detection threshold and $r_{i}$ is given by

$$
\begin{aligned}
& r_{1}=\eta P_{\mathrm{S}}\left[D+P_{\mathrm{A}}\right] \beta h+N_{\mathrm{DCR}} \\
& r_{0}=\eta P_{\mathrm{S}}\left[D-P_{\mathrm{A}}\right] \beta h+N_{\mathrm{DCR}}
\end{aligned},
$$

with $\eta=C_{\mathrm{PDE}} / E_{\mathrm{p}}$ where $C_{\mathrm{PDE}}$ denotes the photon detection efficiency, $E_{\mathrm{p}}$ is the photon energy and $N_{\mathrm{DCR}}$ is the dark count ratio of SPAD. Then, the average number of counted photons when the symbol $i$ is transmitted, i.e., 0 or 1 , can be written as,

$$
\begin{aligned}
& \lambda_{1}:=c_{1} \beta+N \\
& \lambda_{0}:=c_{0} \beta+N
\end{aligned} .
$$

Firstly, to measure the communication performance, we use the throughput that is defined as

$$
T=(1-\mathcal{P}) H_{\mathrm{p}} R,
$$

where $R=\frac{1}{T_{\mathrm{b}}}$ is the transmission rate and satisfies $R \leq$ $B$ with $B$ is the bandwidth, $H_{\mathrm{p}}=-\sum_{i} p_{i} \log _{2}\left(p_{i}\right)$ is the entropy and $\mathcal{P}$ denotes the AQ SPAD error probability which is similar to that of a SPAD in absence of dead time, but with a reduced quantum efficiency. With $\lambda_{\text {th }}<k_{\max }$, it is expressed as follows for a given channel $h$ [11],

$$
\mathcal{P}=p_{0}\left(1-\sum_{k=0}^{\lambda_{\text {th }}} \psi\left(k, \lambda_{0}\right)\right)+p_{1}\left(\sum_{k=0}^{\lambda_{\text {th }}} \psi\left(k, \lambda_{1}\right)\right) .
$$

Using the upper incomplete gamma function $\Gamma(x, y)$ and the gamma function $\Gamma(n)$, (13) can be written as follows,

$$
\mathcal{P}=p_{0}\left(1-\frac{\Gamma\left(\lambda_{\mathrm{th}}+1, \lambda_{0}\right)}{\Gamma\left(\lambda_{\mathrm{th}}+1\right)}\right)+p_{1}\left(\frac{\Gamma\left(\lambda_{\mathrm{th}}+1, \lambda_{1}\right)}{\Gamma\left(\lambda_{\mathrm{th}}+1\right)}\right),
$$

where, assuming that $r_{0} \gg 1$ or $\tau \ll T_{\mathrm{b}}$, the optimum threshold $\lambda_{\mathrm{th}}$ of the maximum a posteriori (MAP) detection employed for OOK demodulation, is as follows,

$$
\lambda_{\mathrm{th}}=\left\lfloor\frac{\left(r_{1}-r_{0}\right) T_{\mathrm{b}}-\left(r_{1}-r_{0}\right) \tau+\ln \left(\frac{p_{0}}{p_{1}}\right)}{\left(r_{1}-r_{0}\right) \tau+\ln \left(\frac{r_{1}}{r_{0}}\right)}\right\rfloor .
$$

Also, in case of equal probability, the detection threshold is expressed as in [11],

$$
\lambda_{\mathrm{th}}=\frac{\left(r_{1}-r_{0}\right) T_{\mathrm{b}}-\left(r_{1}-r_{0}\right) \tau}{\left(r_{1}-r_{0}\right) \tau+\ln \left(\frac{r_{1}}{r_{0}}\right)} .
$$


Secondly, we express the received solar cell signal as [9], $y_{\mathrm{SC}}(t)=\underbrace{r h(1-\beta) P_{\mathrm{s}} D+\mathcal{I}}_{I_{\mathrm{DC}}}+\underbrace{r h(1-\beta) P_{\mathrm{s}} m(t)-\mathcal{I}+N_{\mathrm{AWGN}}(t)}_{I_{\mathrm{AC}}(t)}$,

where $r$ is the solar cell responsivity, $N_{\mathrm{AWGN}}(t)$ is the Additive White Gaussian Noise (AWGN) with zero mean and $\sigma_{\mathrm{N}}^{2}$ variance, $I_{\mathrm{DC}}$ is the $\mathrm{DC}$ component used by the solar cell to harvest energy, $I_{\mathrm{AC}}(t)$ is the $\mathrm{AC}$ component of the received signal and $\mathcal{I}$ is found such that the average of the AC component, vanishes, i.e., $\mathcal{I}$ is found to be

$$
\mathcal{I}=r h(1-\beta) P_{\mathrm{s}}\left(p_{1} P_{\mathrm{A}}-p_{0} P_{\mathrm{A}}\right) .
$$

Thus, the solar cell harvested energy is given by [9]

$$
E=C_{\mathrm{FF}} I_{\mathrm{DC}} V_{\mathrm{t}} \ln \left(1+\frac{I_{\mathrm{DC}}}{I_{0}}\right),
$$

where $C_{\mathrm{FF}}$ is the fill factor, $V_{\mathrm{t}}$ is the thermal voltage and $I_{0}$ is the solar cell dark saturation current.

\section{ENERGY-AWARE UNDERWATER SLIPT SYSTEM DESIGN}

In this section, we define the energy-aware design problem of the proposed SLIPT system, study the feasibility problem and find the optimal power splitting and detection threshold.

\section{A. Problem definition}

We define the energy-aware design problem as follows,

$$
\begin{array}{cl}
\max _{\beta, \lambda_{\mathrm{th}}} & E(\beta) \\
\text { s.t. } & T\left(\lambda_{\mathrm{th}}, \beta\right) \geq \widetilde{T_{\mathrm{th}}}, \\
& \mathcal{P}\left(\lambda_{\mathrm{th}}, \beta\right) \leq \mathcal{P}_{\mathrm{t}}, \\
& 0<\beta<1, \\
& 2 \leq \lambda_{\mathrm{th}} \leq \lambda_{\mathrm{th}_{\max }} .
\end{array}
$$

where $\widetilde{T_{\mathrm{th}}}$ is the minimum throughput required for the communication link, $\mathcal{P}_{\mathrm{t}}$ is the maximum allowable bit error rate, and $\lambda_{\mathrm{th}_{\max }}=k_{\max }-2$ is the maximum detection threshold.

To simplify the problem, we first express (20.c) equivalently in terms of the throughput as $T\left(\lambda_{\mathrm{th}}, \beta\right) \geq T_{\min }$ where $T_{\min }=$ $\left(1-\mathcal{P}_{\mathrm{t}}\right) R H_{\mathrm{p}}$ presenting the minimum link reliability avoiding trivial scenarios. Then, we combine it with (20.b) introducing the new equivalent constraint $T\left(\lambda_{\mathrm{th}}, \beta\right) \geq T_{\mathrm{th}}$ where $T_{\mathrm{th}}=$ $\max \left(T_{\min }, \widetilde{T_{\mathrm{th}}}\right)$ with $T_{\min }<T_{\mathrm{th}}<T_{\max }$ and $T_{\max }=R H_{\mathrm{p}}$ describes the maximum throughput limit for the system. The new constraint can be rewritten using (12) and (14) as,

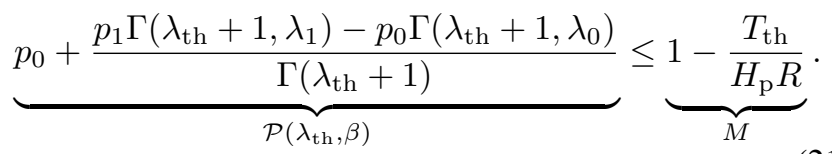

Hence, the simplified optimization problem is

$$
\begin{array}{cl}
\max _{\beta, \lambda_{\mathrm{th}}} & E(\beta) \\
\text { s. t. } & \mathcal{P}\left(\lambda_{\mathrm{th}}, \beta\right) \leq M, \\
& T_{\min }<T_{\mathrm{th}}<T_{\max }, \\
& 0<\beta<1, \\
& 2 \leq \lambda_{\mathrm{th}} \leq \lambda_{\mathrm{th}_{\max }} .
\end{array}
$$

The problem in (22) is a mixed integer nonlinear optimization problem. Thus, for each $\lambda_{\mathrm{th}}$, we define and solve the following inner optimization problem to find the corresponding optimal $\beta$, then the optimal solution is determined by going through all $\lambda_{\text {th }}$ values and finding the $\lambda_{\text {th }}$ that maximizes the harvested energy.

$$
\begin{aligned}
\left(P_{\text {inner }}\right): \max _{\beta} & E(\beta) \\
\text { s.t. } \quad & \mathcal{P}(\beta) \leq M \\
& T_{\min }<T_{\text {th }}<T_{\max }, \\
& 0<\beta<1 .
\end{aligned}
$$

Unfortunately, $P_{\text {inner }}$ problem is not convex problem, where $E(\beta)$ is not a concave function in $\beta$ and $\mathcal{P}$ is not a convex function in $\beta$. However, we analyze the behavior of both functions in the following sections to find the optimal solution.

\section{B. Optimal power splitting algorithm}

First, we find that $E(\beta)$ is a decreasing convex function for all values of $\beta \in[0,1]$. The decreasing behavior motivated us to analyze the performance of $\mathcal{P}(\beta)$ to check whether it is increasing, decreasing or has both behaviors. Thus, we derive the first derivative of of $\mathcal{P}(\beta)$, which is found to be

$$
\frac{\partial \mathcal{P}(\beta)}{\partial \beta}=\frac{c_{0} \lambda_{0}^{\lambda_{\text {th }}} e^{-\lambda_{0}}-p_{1}\left(c_{0} \lambda_{0}^{\lambda_{\text {th }}} e^{-\lambda_{0}}+c_{1} \lambda_{1}^{\lambda_{\text {th }}} e^{-\lambda_{1}}\right)}{\Gamma\left(\lambda_{\text {th }}+1\right)} .
$$

After studying (24), we find four possible scenarios depending on the value of $p_{1}, \mathcal{P}(\beta)$ can either have a stationary point (it is decreasing/increasing or increasing/decreasing), it is increasing, or decreasing. However, some of these scenarios do not yield to a feasible optimization problem. Thus, by examining the constraint $\mathcal{P}(\beta) \leq M$, we derive the problem feasibility and solution in each scenario. In fact, the intersection between $\mathcal{P}(\beta)$ and $M$ is necessary to have a feasible solution that yields into a reliable communication system. For example, if $\mathcal{P}(\beta)<M$ for all $\beta \in[0,1]$, regardless of the behaviour of $\mathcal{P}(\beta)$, the solution is $\beta=0$ which is a trivial system. Hence, it is imperative to find the solution $\mathcal{P}(\beta)=M$ for each scenario, in order to define the feasibility region, which depends on the value of $T_{\mathrm{th}}$. Then, the problem solution becomes the minimum value of $\beta$ in the feasible domain since the objective function is a decreasing function in $\beta$.

Let $\beta_{s}$ be the solution of $\mathcal{P}(\beta)=M$ when $\mathcal{P}(\beta)$ doesn't have a stationary point. If $\mathcal{P}(\beta)$ has a stationary point $\beta_{\mathrm{d}}$; then, let $\beta_{s 1}$ and $\beta_{s 2}$ be the solutions of $\mathcal{P}(\beta)=M$ with $\beta_{s 1}<\beta_{s 2}$. Table (I) outlines the feasible scenarios with their behaviors given as conditions on $p_{1}$ and their feasibility ranges as conditions on $T_{\mathrm{th}}$, as well as the solution of the optimization problem where $p_{\mathrm{b} 1}=\frac{c_{0}}{c_{0}+c_{1}}$ and $p_{\mathrm{b} 2}=$ $\left(\left(\frac{c_{1}}{c_{0}}\right)\left(\frac{c_{1}+N}{c_{0}+N}\right)^{\lambda_{\mathrm{th}}} e^{-\left(c_{1}-c_{0}\right)}+1\right)^{-1}$.

Using these conditions, we propose our algorithm (Algorithm 1) that describes the instructions to solve the whole optimization problem in (20) with respect to the power splitting ratio $\beta$ and the detection threshold $\lambda_{\mathrm{th}}$ for given $p_{1}$ and $T_{\mathrm{th}}$.

\section{NuMERICAL RESULTS}

In this section, we present our numerical results. Table (II) summarises the system parameters including the trans- 


\begin{tabular}{|c|c|c|c|c|}
\hline Case & Condition on $p_{1}$ & Condition on $T_{\mathrm{th}}$ & Feasibility range & Solution \\
\hline (1) & $\max \left(p_{\mathrm{b} 1}, p_{\mathrm{b} 2}\right)<p_{1}<1$ & $H_{\mathrm{p}} R(1-\mathcal{P}(0))<T_{\mathrm{th}}<H_{\mathrm{p}} R(1-\mathcal{P}(1))$ & $\beta_{\mathrm{S}}<\beta<1$ & $\beta=\beta_{\mathrm{s}}$ \\
\hline (2) & \multirow[b]{2}{*}{$p_{\mathrm{b} 1}<p_{1}<p_{\mathrm{b} 2}$} & $\max \left(H_{\mathrm{p}} R(1-\mathcal{P}(0)), H_{\mathrm{p}} R(1-\mathcal{P}(1))\right)<T_{\mathrm{th}}<H_{\mathrm{p}} R\left(1-\mathcal{P}\left(\beta_{\mathrm{d}}\right)\right)$ & $\beta_{\mathrm{s} 1}<\beta<\beta_{\mathrm{s} 2}$ & $\beta=\beta_{\mathrm{s} 1}$ \\
\hline (3) & & $H_{\mathrm{p}} R(1-\mathcal{P}(0))<T_{\mathrm{th}}<H_{\mathrm{p}} R(1-\mathcal{P}(1))$ & $\beta_{\mathrm{s} 1}<\beta<1$ & $\beta=\beta_{\mathrm{s} 1}$ \\
\hline (4) & \multirow{2}{*}{$p_{\mathrm{b} 2}<p_{1}<p_{\mathrm{b} 1}$} & $H_{\mathrm{p}} R\left(1-\mathcal{P}\left(\beta_{\mathrm{d}}\right)\right)<T_{\mathrm{th}}<\min \left(H_{\mathrm{p}} R(1-\mathcal{P}(0)), H_{\mathrm{p}} R(1-\mathcal{P}(1))\right)$ & $\begin{array}{l}0<\beta<\beta_{\mathrm{s} 1} \\
\beta_{\mathrm{s} 2}<\beta<1\end{array}$ & $\beta=\beta_{\mathrm{s} 1}$ \\
\hline (5) & & $H_{\mathrm{p}} R(1-\mathcal{P}(0))<T_{\mathrm{th}}<H_{\mathrm{p}} R(1-\mathcal{P}(1))$ & $\beta_{\mathrm{s} 2}<\beta<1$ & $\beta=\beta_{\mathrm{s} 2}$ \\
\hline
\end{tabular}

TABLE I: Summary of the resolution

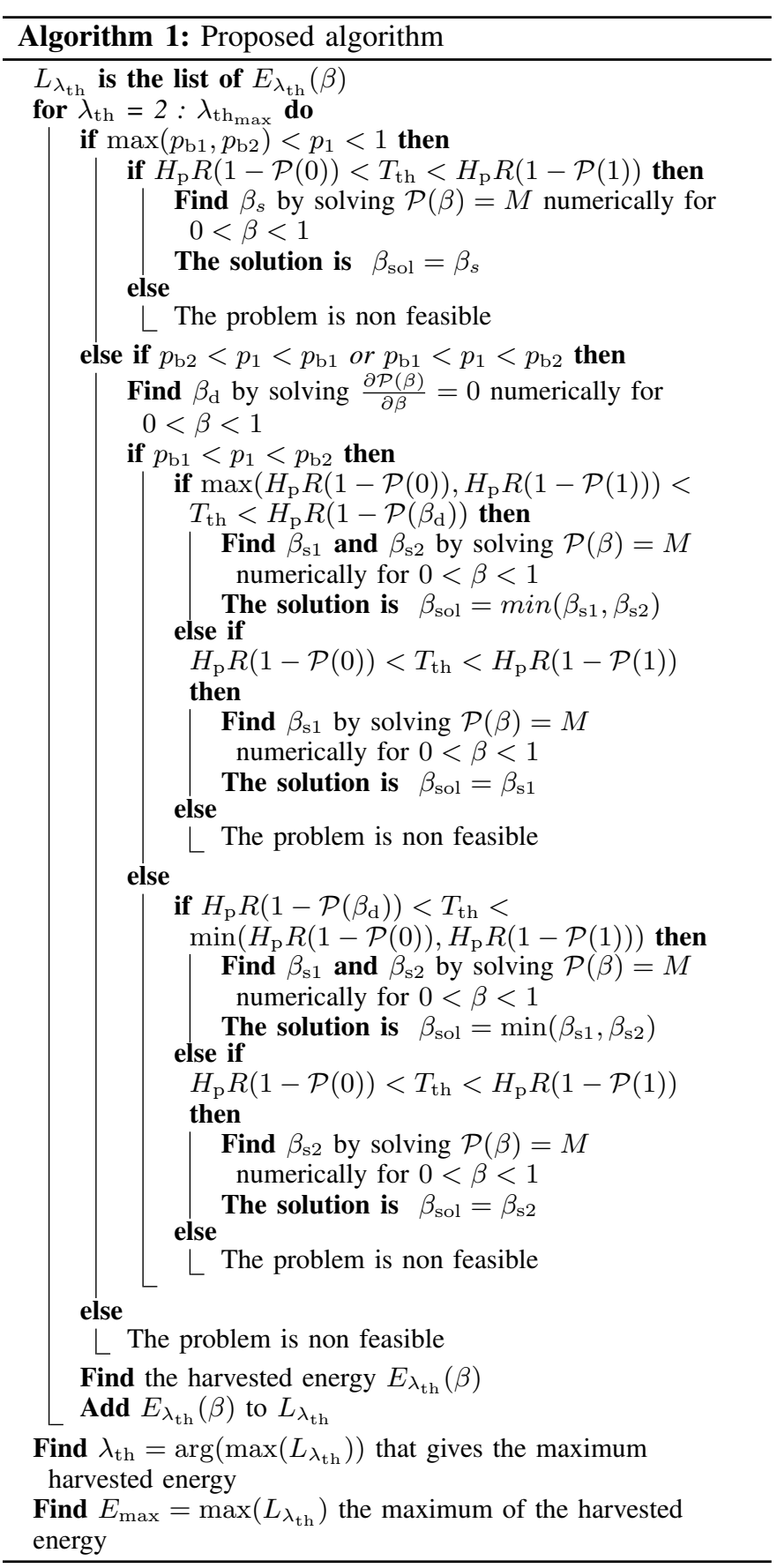

mitter and the receiver (SPAD and solar cell) specifications. Also, to carry out the simulations we assume a bit duration of $T_{\mathrm{b}}=10^{-7} \mathrm{~s}$ and a correction coefficient of $T=0.21$ [9] as well as a clear ocean as water type with extinction coefficient $c_{\mathrm{ex}}=0.15$ and bubble level of $B L=16.5 \mathrm{~L} / \mathrm{min}$ where the EGG parameters [14] are $(\omega, \mu, a, b, c)=(0.4951,0.1368,0.0161,3.2033,82.1030)$.

\begin{tabular}{|l|l|}
\hline Parameter & Value \\
\hline Wavelength $(\lambda)$ & $530 \mathrm{~nm}[9]$ \\
\hline Laser optical power $\left(P_{\mathrm{S}}\right)$ & $20 \mathrm{~W} / \mathrm{A}$ \\
\hline DC bias $(D)$ & $35 \mathrm{~mA}$ \\
\hline Minimum input bias current $\left(I_{\mathrm{L}}\right)$ & $25 \mathrm{~mA}[9]$ \\
\hline Maximum input bias current $\left(I_{\mathrm{H}}\right)$ & $45 \mathrm{~mA}[9]$ \\
\hline Responsivity of solar cell $(r)$ & $0.4 \mathrm{~A} / \mathrm{W}[9]$ \\
\hline Fill factor of solar cell $\left(C_{\mathrm{FF}}\right)$ & $0.75[9]$ \\
\hline Dark saturation current of solar cell $\left(I_{0}\right)$ & $10^{-9} \mathrm{~A}[9]$ \\
\hline Thermal voltage of solar cell $\left(V_{\mathrm{t}}\right)$ & $25 \mathrm{mV}[9]$ \\
\hline Photon detection efficiency of SPAD $\left(C_{\mathrm{PDE}}\right)$ & $0.5[15]$ \\
\hline Dark count ratio of SPAD $\left(N_{\mathrm{DCR}}\right)$ & $50 \mathrm{cps}[15]$ \\
\hline Receiver aperture diameter $\left(D_{\mathrm{R}}\right)$ & $20 \mathrm{~cm}[9]$ \\
\hline Full width transmitter beam divergence angle $(\theta)$ & $6^{\circ}[9]$ \\
\hline
\end{tabular}

TABLE II: System parameters

Additionally, we suppose that the BER target is $\mathcal{P}_{\mathrm{t}}=0.1$ to guarantee a minimum link reliability.

Firstly, the optimization problem is feasible only under certain conditions on the values of the probability $p_{1}$ and the throughput $T_{\mathrm{th}}$. Hence, to properly choose them, it is essential to study the feasibility region. The latter can be obtained using Algorithm 1 where the "if" instructions correspond to the conditions on $p_{1}$ and $T_{\mathrm{th}}$. These conditions depend on the system parameters, namely the distance and the dead time $\tau$. In fact, the feasibility range is broad for small dead times and long distances while, it shrinks for shorter link ranges and higher dead times. Fig.1 illustrates the feasibility domain for distance $d=50 \mathrm{~m}$ and dead time $\tau=2.10^{-13} \mathrm{~s}$ under a normalised underwater channel with turbulence coefficient $\alpha=1$. This figure provides us with a general idea about the feasible range of $T_{\text {th }}$ values for each $p_{1}$ and displays the feasible region (in blue color), which is limited by lower and upper bounds. While the lower limit is explained by the value of $T_{\text {min }}$, for each $p_{1}$, ensuring a minimal level of transmission quality, the upper boundary is defined by $T_{\max }$ representing the maximum throughput that the system can achieve.

Secondly, the effect of the dead time and the link range on the problem feasibility and the maximum harvested energy is studied as shown in Fig. 2 for $p_{1}=0.5, T_{\text {th }}=9.5 \mathrm{Mbps}$, and different dead time values $\tau=2.10^{-9} s, 2.10^{-11} s$ and $2.10^{-13} s$. The impact of the dead time on the link range is observed by the problem's feasibility in Fig. 2. For example, for $d=50 \mathrm{~m}$, SPADs with $\tau=2.10^{-9} s$ or higher can not support the required throughput and BER performance. Such behavior occurs due to the dead time limitation imposed on the SPAD's photon counting process, which determines the link reliability, where the detection threshold should satisfy $\lambda_{\text {th }}<k_{\max }=\left\lfloor T_{\mathrm{b}} / \tau\right\rfloor+1$. Particularly, for short distances the average photon arrival rate is very large which restricts the SPAD's ability to decode the data, whereas, it is limited for longer distances, due to the channel effect, enabling the SPAD to properly decode the transmitted symbols. Also, Fig.2 shows that adopting a combined receiver enables the system 


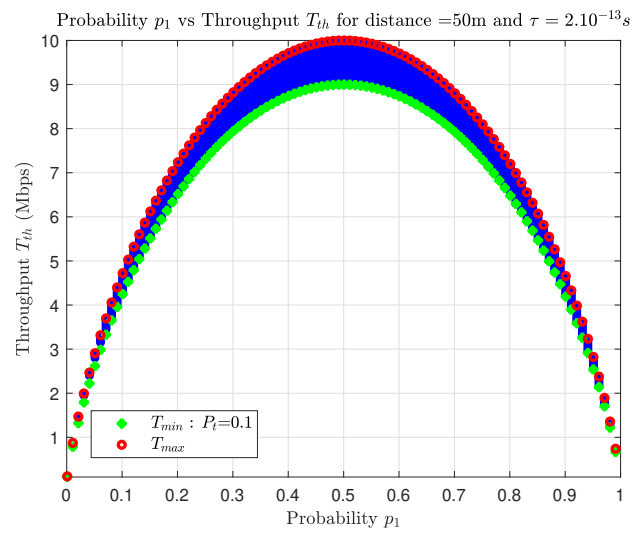

Fig. 1: Feasibility region (in blue color) in terms of probability $p_{1}$ and throughput $T_{\text {th }}$ for turbulence coefficient $\alpha=1$, dead time $\tau=2 \cdot 10^{-13} \mathrm{~s}$ and distance $d=50 \mathrm{~m}$.

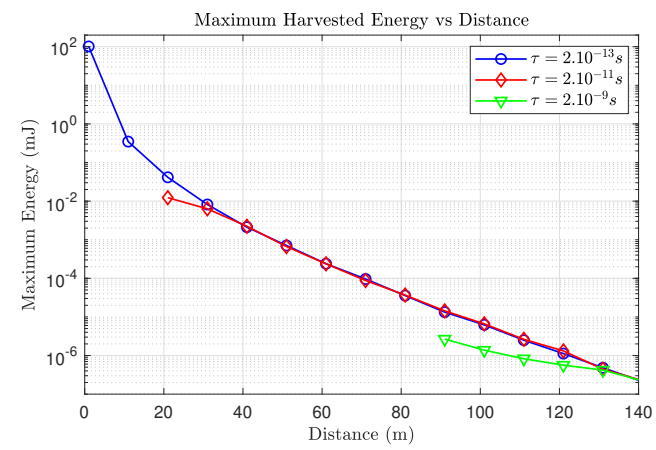

Fig. 2: Average maximum harvested energy according to distance for different dead times with $p_{1}=0.5$ and $T_{\mathrm{th}}=$ $9.5 \mathrm{Mbps}$, computed numerically using $n_{\alpha}=1000$ sample.

to achieve a throughput of $9.5 \mathrm{Mbps}$ over long link ranges (up to $140 \mathrm{~m}$ ), but with a limited amount of harvested energy. So, from energy perspective, it is more interesting to observe its behavior for shorter distances.

Finally, Fig. 3 illustrates the behavior of harvested energy with respect to the communication range and different values of $p_{1}$ and $T_{\mathrm{th}}$ for $\tau=2.10^{-13} \mathrm{~s}$. We notice that employing high $p_{1}$ values $\left(p_{1}=0.9\right.$ or 0.8$)$ results in greater energy harvesting at the cost of having low throughput performance. Meanwhile, better communication service can be reached with a small reduction in the amount of harvested energy when adopting probabilities around $0.5\left(p_{1}=0.5\right.$ or 0.4$)$. Moreover, under the same harvested energy, the best throughput is achieved with equal probability $\left(p_{1}=0.5\right)$. Therefore, we observe a trade-off between the harvested energy and the achievable throughput performance.

\section{CONClusion}

In this paper, we proposed an underwater SLIPT system based on a combined receiver composed of a solar cell and a SPAD to perform energy harvesting and information decoding simultaneously. To achieve this goal, we employed the power splitting SLIPT method and maximized the harvested energy to the power splitting factor while guaranteeing a minimal communication performance. After studying the optimization problem, we determined the problem feasibility and proposed

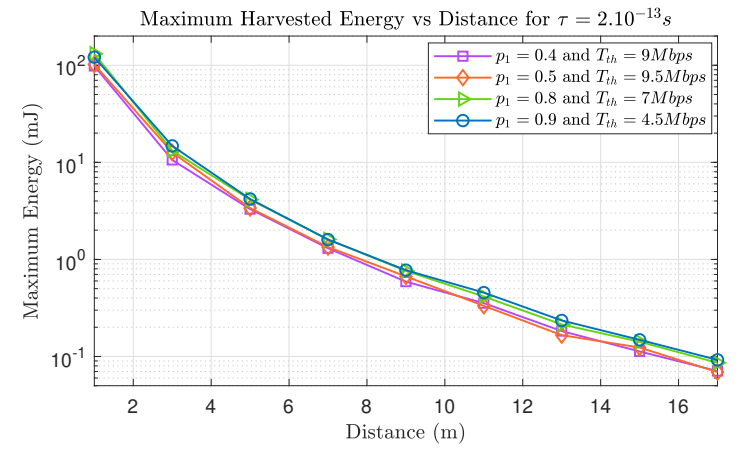

Fig. 3: Average Maximum harvested energy according to distance for $\tau=2.10^{-13} s$ and different $p_{1}$ and $T_{\mathrm{th}}$.

an algorithm that finds the solution. Our results showed that employing a SPAD with the solar cell at the receiver side yields throughput performance improvement; however, the performance can be limited by the SPAD dead time, which can be mitigated in future work by employing SPAD arrays.

\section{REFERENCES}

[1] A. Celik, N. Saeed, B. Shihada, T. Y. Al-Naffouri, and M.-S. Alouini, "A software-defined opto-acoustic network architecture for internet of underwater things," IEEE Commun. Mag., vol. 58, no. 4, pp. 88-94, Apr. 2020.

[2] Z. Zeng, S. Fu, H. Zhang, Y. Dong, and J. Cheng, "A survey of underwater optical wireless communications," IEEE Commun. Surveys Tuts., vol. 19, no. 1, pp. 204-238, 2016.

[3] H. Kaushal and G. Kaddoum, "Underwater optical wireless communication," IEEE Access, vol. 4, pp. 1518-1547, 2016.

[4] B. Shihada, O. Amin, C. Bainbridge, S. Jardak, O. Alkhazragi, T. K. $\mathrm{Ng}$, B. Ooi, M. Berumen, and M.-S. Alouini, "Aqua-Fi: Delivering internet underwater using wireless optical networks," IEEE Commun. Mag., vol. 58, no. 5, pp. 84-89, May 2020.

[5] P. D. Diamantoulakis, G. K. Karagiannidis, and Z. Ding, "Simultaneous lightwave information and power transfer (SLIPT)," IEEE Trans. Green Commun. Netw., vol. 2, no. 3, pp. 764-773, Sept. 2018.

[6] A. M. Abdelhady, O. Amin, B. Shihada, and M.-S. Alouini, "Spectral efficiency and energy harvesting in multi-cell SLIPT systems," IEEE Trans. Wireless Commun., vol. 19, no. 5, pp. 3304-3318, May 2020.

[7] J. I. de Oliveira Filho, A. Trichili, B. S. Ooi, M.-S. Alouini, and K. N. Salama, "Toward self-powered Internet of underwater things devices," IEEE Commun. Mag., vol. 58, no. 1, pp. 68-73, Jan. 2020.

[8] M. Kong, B. Sun, R. Sarwar, J. Shen, Y. Chen, F. Qu, J. Han, J. Chen, H. Qin, and J. Xu, "Underwater wireless optical communication using a lens-free solar panel receiver," Optics Commun., vol. 426, pp. 94-98, Nov. 2018.

[9] S. Ghasvarianjahromi, M. Karbalayghareh, P. D. Diamantoulakis, G. K. Karagiannidis, and M. Uysal, "Simultaneous lightwave information and power transfer in underwater visible light communications," in IEEE 30th PIMRC, 2019, pp. 1-6.

[10] T. Shafique, O. Amin, M. Abdallah, I. S. Ansari, M.-S. Alouini, and K. Qaraqe, "Performance analysis of single-photon avalanche diode underwater VLC system using ARQ," IEEE Photon. J, vol. 9, no. 5, pp. 1-11, Oct. 2017.

[11] E. Sarbazi, M. Safari, and H. Haas, "Statistical modeling of singlephoton avalanche diode receivers for optical wireless communications," IEEE Trans. Commun., vol. 66, no. 9, pp. 4043-4058, Sept. 2018.

[12] A. Elzanaty and M.-S. Alouini, "Adaptive coded modulation for IM/DD free-space optical backhauling: A probabilistic shaping approach," IEEE Trans. Commun., vol. 68, no. 10, pp. 6388-6402, Oct. 2020.

[13] M. Elamassie, F. Miramirkhani, and M. Uysal, "Performance characterization of underwater visible light communication," IEEE Trans. Commun., vol. 67, no. 1, pp. 543-552, Jan. 2019.

[14] E. Zedini, H. M. Oubei, A. Kammoun, M. Hamdi, B. S. Ooi, and M.-S. Alouini, "Unified statistical channel model for turbulence-induced fading in underwater wireless optical communication systems," IEEE Commun. Mag., vol. 67, no. 4, pp. 2893-2907, April 2019.

[15] SPCM-AQRH, Single Photon Counting Module, Excelitas Technologies, 2020, Datasheet, Photon Detection. [Online]. Available: https://www. excelitas.com/product/spcm-aqrh 